\title{
How Proofs are Prepared at Camelot
}

\author{
[Extended Abstract $]^{\dagger}$
}

\author{
Andreas Björklund \\ Department of Computer Science \\ Lund University \\ Lund, Sweden \\ andreas.bjorklund@yahoo.se
}

\author{
Petteri Kaski \\ Helsinki Institute for Information Technology HIIT \\ Department of Computer Science \\ Aalto University \\ Helsinki, Finland \\ petteri.kaski@aalto.fi
}

\begin{abstract}
We study a design framework for robust, independently verifiable, and workload-balanced distributed algorithms working on a common input. The framework builds on recent noninteractive Merlin-Arthur proofs of batch evaluation of Williams [31st IEEE Colloquium on Computational Complexity (CCC'16, May 29-June 1, 2016, Tokyo), to appear] with the basic observation that Merlin's magic is not needed for batch evaluation: mere Knights can prepare the independently verifiable proof, in parallel, and with intrinsic errorcorrection.

As our main technical result, we show that the $k$-cliques in an $n$-vertex graph can be counted and verified in pernode $O\left(n^{(\omega+\epsilon) k / 6}\right)$ time and space on $O\left(n^{(\omega+\epsilon) k / 6}\right)$ compute nodes, for any constant $\epsilon>0$ and positive integer $k$ divisible by 6 , where $2 \leq \omega<2.3728639$ is the exponent of square matrix multiplication over the integers. This matches in total running time the best known sequential algorithm, due to Nešetřil and Poljak [Comment. Math. Univ. Carolin. 26 (1985) 415-419], and considerably improves its space usage and parallelizability. Further results (only partly presented in this extended abstract) include novel algorithms for counting triangles in sparse graphs, computing the chromatic polynomial of a graph, and computing the Tutte polynomial of a graph.
\end{abstract}

\footnotetext{
${ }^{*}$ The research leading to these results has received funding from the Swedish Research Council grant VR 2012-4730 "Exact Exponential-Time Algorithms" (A.B.) and the European Research Council under the European Union's Seventh Framework Programme (FP/2007-2013) / ERC Grant Agreement 338077 "Theory and Practice of Advanced Search and Enumeration" (P.K.). Work done in part while the authors were visiting the Simons Institute for the Theory of Computing.

${ }^{\dagger}$ A full version of this paper is available at http://arxiv.org/ abs/1602.01295
}

Permission to make digital or hard copies of all or part of this work for personal or classroom use is granted without fee provided that copies are not made or distributed for profit or commercial advantage and that copies bear this notice and the full citation on the first page. Copyrights for components of this work owned by others than the author(s) must be honored. Abstracting with credit is permitted. To copy otherwise, or republish, to post on servers or to redistribute to lists, requires prior specific permission and/or a fee. Request permissions from permissions@acm.org.

PODC'16, July 25-28, 2016, Chicago, IL, USA

(C) 2016 Copyright held by the owner/author(s). Publication rights licensed to ACM. ISBN 978-1-4503-3964-3/16/07 . \$15.00

DOI: http://dx.doi.org/10.1145/2933057.2933101

\section{Keywords}

batch evaluation; noninteractive proof; parameterized clique; Reed-Solomon; vector parallel; verification

\section{INTRODUCTION}

\subsection{A scene of distress and relief at Camelot}

Picture $K$ Knights seated around the Round Table, distressed. At the center of the table stands the Input. The Knights have been tasked to prepare a Proof about the virtues of the input, and to make extreme haste: a proof must be prepared in time $T / K$, where $T$ is the fastest time known in all of Britannia for any single soul to reveal such subtle virtues, let alone give uncontestable proof thereof.

Nigh impossible is the task in fact, for the Lady Morgana has enchanted many a poor Knight with her cunning dark magic, yet virtues must be revealed and proof thereof prepared. And not just any proof, but one that any lone soul can check, in time $T / K$ and with a few tosses of a fair coin, and next to never be convinced if foul virtues it claims.

However, not all is lost, for the Knights recall the teachings of the wizard Merlin ${ }^{1}$ and his powerful proofs that King Arthur so treasures. So the Knights agree to together evaluate in batch, and to individually decode-and-check for signs of the darkest of magic...

\subsection{A template for community computation over common input}

Building on Merlin-Arthur proofs 2] of batch evaluation of Williams 25, this paper documents a "Camelot template" for verifiable distributed computation that is robust against adversarial byzantine failures at the nodes and produces a static, independently verifiable proof that the computation succeeded. In essence, we observe that the act of preparing a proof in the Williams framework is magicless and robust against errors. Our contribution is in particular to show that for a number of problems such robust and correctness-proofproducing algorithms essentially match in total resource consumption the best known sequential algorithm for solving the problem.

We assume the following model for $K$ distributed compute nodes with unique node identifiers. Each compute node has access to its own source of randomness that is independent

\footnotetext{
${ }^{1}$ Alas, the good wizard is taking a vacation, hence the distress at Camelot.
} 
of the other nodes. The nodes are joined by a network that supports broadcast operations that take place (a) initially to all the nodes simultaneously, and, (b) after each node has finished computing, one short broadcast from the node to all the nodes. Here (a) is to broadcast the complete input (the problem instance) to the nodes, and (b) is to broadcast the encoded proof fragment that a node has computed to the other nodes. We assume sufficient synchronization exists in the network to coordinate the broadcasts so that each node knows when phases (a) and (b) are complete. Apart from this end-of-phase synchronization, broadcast operations may fail arbitrarily. For example, a broadcast may fail so that nodes may receive different and/or corrupt fragments in (b), or in (a) a node may receive an incorrect input that looks like a correct input. Furthermore, the nodes do not seek consensus. That is, at the end of phase (b), each node executes its own, individual check on the data it received and decides (probabilistically, using the random source at the node) whether to accept or reject the proof produced by the nodes.

Our subsequent algorithms guarantee that any entity (a compute node or an outside party) that has the correct input and a sufficient fraction of correctly encoded proof fragments will decode the correct proof and accept it. Furthermore, any entity that has the correct input will reject any incorrect proof presented to it (in encoded or decoded form) with high probability that can be controlled after the proof is presented for verification. In particular, any node with a correct proof can convince other nodes that it has a correct proof, but such subsequent consensus-seeking within the community after the algorithm has been executed is not within scope of the present paper.

\subsection{How Camelot prepares, corrects, and checks a proof}

We now review the Williams framework 25] and interpret it as a template for distributed computing in the assumed model with $K$ computes nodes. The key idea is to task the $K$ nodes to make evaluations of a univariate polynomial

$$
P(x)=p_{0}+p_{1} x+p_{2} x^{2}+\ldots+p_{d} x^{d}(\bmod q),
$$

where $q$ is a prime that we assume each node can easily compute by looking at the input that it has received from the network. We also assume that each node can easily compute an upper bound for $d$ from the input.

The polynomial $P(x)$ has been carefully constructed so that (a) it reveal ${ }^{2}$ the desired properties of the input, and (b) the $d+1$ coefficients $p_{0}, p_{1}, \ldots, p_{d} \in \mathbb{Z}_{q}$ constitute a probabilistically verifiable proof that these properties have been correctly computed. In fact, the latter property is intrinsic to the template.

1. Proof preparation, in distributed encoded form. For a prime $q$ and an integer $e$ with $d+1 \leq e \leq q$, we task the $K$ nodes to compute the sequence of $e$ evaluations

$$
P(0), P(1), \ldots, P(e-1) \quad(\bmod q)
$$

so that each node is responsible for about $e / K$ evaluations, and broadcasts its evaluations to the other nodes once they are ready. This sequence of evaluations is, by definition, an

\footnotetext{
${ }^{2}$ Possibly requiring iteration and postprocessing, e.g., the use of multiple distinct primes $q$ and the Chinese Remainder Theorem to reconstruct large integers.
}

encoding of the proof in the classical nonsystematic ReedSolomon code 23 (see \$2). From the perspective of community computation, this is a serendipitous property:

2. Error-correcting and decoding the proof. Since the nodes are tasked to produce entries in a Reed-Solomon codeword, each node can, on its own, run a fast Reed-Solomon decoder 15. (see \$2) on the entries it has received from the network to recover both the actual proof $p_{0}, p_{1}, \ldots, p_{d} \in \mathbb{Z}_{q}$ and the evaluations $P(0), P(1), \ldots, P(e-1) \in \mathbb{Z}_{q}$ that failed, that is, the error locations identified by the decoder. In precise terms, to recover the correct proof using, e.g., the Gao 15] decoder, a node needs at least $(e+d+1) / 2$ correct evaluations among the $e$ evaluations for decoding to succeed at a node (see $\$ 2.2$.

3. Checking a putative proof for correctness. Each node (or any other entity) that has available the correct input and a sequence $\tilde{p}_{0}, \tilde{p}_{1}, \ldots, \tilde{p}_{d} \in \mathbb{Z}_{q}$ can, probabilistically, check whether the sequence is in fact the correct proof $p_{0}, p_{1}, \ldots, p_{d}$ $\in \mathbb{Z}_{q}$. This check is executed locally without any interaction with the network. All a node has to do is to select a uniform random integer $x_{0} \in \mathbb{Z}_{q}$ and accept the proof if and only if

$$
P\left(x_{0}\right)=\sum_{j=0}^{d} \tilde{p}_{j} x_{0}^{j} \quad(\bmod q)
$$

holds, at the cost of one evaluation of $P(x)$. (That is, the verifier executes the same algorithm that the nodes use for preparing the proof to obtain the left-hand side of (2); the right-hand side can be computed from $\tilde{p}_{0}, \tilde{p}_{1}, \ldots, \tilde{p}_{d} \in \mathbb{Z}_{q}$ using Horner's rule.) If the sequence $\tilde{p}_{0}, \tilde{p}_{1}, \ldots, \tilde{p}_{d}$ is the correct proof, the verifier always accepts. If the sequence is incorrect, it follows immediately from the fundamental theorem of algebra that $P\left(x_{0}\right) \neq \sum_{j=0}^{d} \tilde{p}_{j} x_{0}^{j}(\bmod q)$ holds with probability at least $1-d / q$. Furthermore, since the verifier knows $q$ and an upper bound for $d$, the verifier can control the probability of accepting an incorrect proof by independent repetitions of the test.

We stress that a node will, with high probability, not accept an incorrect proof even if arbitrary failures occur in the network and at the other nodes. Furthermore, assuming the failures are not extensive (remain below the decoding threshold) a correct proof will be decoded and accepted at each correctly functioning node that has the correct input. We refer to e.g. Tiwari et al. 24 for recent empirical data why robustness and proofs-of-correctness are increasingly desirable goals in algorithm design.

\subsection{Optimality relative to best known sequential algorithms}

Ideally, a Camelot algorithm should match in total running time the worst-case running time of the best known sequential algorithm for solving the problem. By design, such a Camelot algorithm not only solves the problem, but does so in parallel, robustly, and gives an independently verifiable proof that it succeeded. What is more, the parallel part of the computation (1) consists of evaluating the same polynomial $P$ at distinct points, making the parallel computations intrinsically workload-balanced and ideal for vectorparallelization (single-instruction multiple-data (SIMD) parallelization) since each evaluation follows the same instruction stream. 
In essence, we seek the following optimal speedup tradeoff for a Camelot design. Assume that the best known sequential algorithm solves the problem essentially in time $T$. Then, an optimal Camelot design for $K$ nodes should run in $E=T / K$ proof construction time and space for each node, and hence $E$ evaluation (proof verification) time for a proof of size $K$, for any $K \leq T^{1 / 2}$. Here the upper bound $T^{1 / 2}$ is intrinsic to the framework since each node receives the whole proof for individual decoding and hence $E \geq K$. (This of course does not rule out variants where a single node does not work with the entire proof.)

In practice, our subsequent algorithm designs will not attain the optimal tradeoff for all problems considered. However, for a number of canonical problems we are able to attain the optimal tradeoff.

\subsection{Our results - two highlights}

This conference abstract highlights two examples of problems where we are able to attain the optimal tradeoff; the full version of this paper gives further highlights and an inventory of earlier algorithm designs that can be put into the Camelot framework.

For ease of exposition, we present our results as nontradeoff versions with the maximum value of $K$ (and hence the fastest wall-clock runtime $E$ to prepare and check the proof) that we can attain, with the understanding that a smooth tradeoff up to the maximum $K$ is possible and can be read off the proofs of our results.

When stating our results, we refer to $K$ as the proof size and $E$ as the (running) time of a Camelot algorithm. Put otherwise, the time $E$ of a Camelot algorithm is the wallclock time to prepare and verify the proof using $K$ compute nodes working in parallel on the common input. The total time (sum of running times of all the nodes) is $E K$. The underlying precise degree $d$ of the proof polynomial $P(x)$ is, up to factors suppressed by asymptotic notation, identically bounded as the stated proof size ${ }^{3}$ The stated proof size also bounds the amount of data the nodes in total broadcast across the network during proof preparation. With asymptotic notation we follow the convention that $\tilde{O}(\cdot)$ suppresses a factor polylogarithmic in the input size and $O^{*}(\cdot)$ suppresses a factor polynomial in the input size.

Each Camelot algorithm defines, as is, a Merlin-Arthur protocol 2], where Merlin's proof has the stated size, and Arthur's verification time is the stated time for the Camelot algorithm.

First highlight: counting small cliques. Our main result is that the problem of counting all the $k$-cliques in a graph admits a Camelot algorithm that attains the optimal tradeoff relative to the best known sequential algorithm. Let us write $2 \leq \omega<2.3728639$ for the exponent of square matrix multiplication over the integers [20].

TheOrem 1. For any constant $\epsilon>0$ and any positive integer $k$ divisible by 6 , the number of $k$-cliques in an $n$-vertex

\footnotetext{
${ }^{3}$ The parameters $e$ and $q$ can be chosen with similar asymptotic bounds as $d$. We choose to omit the details of these parameters to keep the statement of our results concise, please consult the proofs for precise bounds on $d$ to enable finetuning of selection of $d+1 \leq e \leq q$ as appropriate for desired error-tolerance (during proof preparation) and soundness guarantee (for proof verification).
}

graph can be computed with a Camelot algorithm that constructs a proof of size $O\left(n^{(\omega+\epsilon) k / 6}\right)$ in time $O\left(n^{(\omega+\epsilon) k / 6}\right)$.

The best known sequential algorithm, due to Nešetřil and Poljak 22] (see also Eisenbrand and Grandoni [10]), runs in time $O\left(n^{(\omega+\epsilon) k / 3}\right)$. The algorithm in Theorem 1 is based on a novel, more space-efficient family of arithmetic circuits for evaluating a particular $\left(\begin{array}{l}6 \\ 2\end{array}\right)$-linear form that enables a very space-efficient design for counting 6 -cliques in a graph.

Second highlight: graph coloring. As our second highlight result, we show that the problem of computing the chromatic polynomial of a given graph admits an optimal Camelot algorithm. Here the best known sequential algorithm runs in $O^{*}\left(2^{n}\right)$ time and $O^{*}\left(1.292^{n}\right)$ space 3,4 .

THEOREM 2. The chromatic polynomial of an n-vertex graph can be computed with a Camelot algorithm that constructs a proof of size $O^{*}\left(2^{n / 2}\right)$ in time $O^{*}\left(2^{n / 2}\right)$.

In particular, Theorem 2 gives an optimal tradeoff. The algorithm in Theorem 2 is a Kronecker-substitution-based polynomial lifting of the parallel partitioning sum-product algorithm of Björklund et al. [4].

Further Camelot designs. Many Camelot algorithms beyond the two highlight designs presented here are possible. We refer to the full version of this conference abstract for a detailed and more gradual exposition.

\subsection{Overview of techniques and earlier work}

The present Camelot framework can be seen as a continuation of two recent works aimed at understanding the finegrained 4 complexity of computational problems and proof systems, initiated in a nondeterministic setting with a deterministic proof verifier by Carmosino et al. 7] and with the subsequent breakthrough by Williams 25] establishing nontrivial quantitative bounds for a randomized verifier. From this perspective, the Camelot framework pursues an even more fine-grained goal by focusing also on the effort of the prover, with the objective of relating the total effort to prepare the proof to the best known sequential algorithms for solving the problem at hand.

We recall the technical preliminaries underlying the framework in $\$ 2$ To design an algorithm in the Camelot framework, all it takes is to come up with the proof polynomial $P$ and a fast evaluation algorithm for $P$. To provide an accessible exposition towards our highlight results, we find it convenient to start (in \$3) with an easier Camelot design and present the Merlin-Arthur protocol of Williams 25 for counting $k$-cliques in an $n$-vertex graph. Viewed as a Camelot algorithm, Williams's design runs in per-node time $\tilde{O}\left(n^{k / 2+1}\right)$ with a proof size of $\tilde{O}\left(n^{k / 2}\right)$, implying a total time that essentially meets the trivial sequential time $O\left(n^{k}\right)$. In particular, Williams's design admits a clean algebrization as the task of verifying a generalized matrix product (cf. Kruskal [18]) using a univariate polynomial extension of Freivalds's classical fingerprinting algorithm for verifying matrix products 13 .

\footnotetext{
${ }^{4}$ Fine-grained in the sense that the objective is to obtain more precise quantitative bounds on resource usage compared with the more traditional coarse-grained and qualitative division between, e.g., polynomial time and exponential time in the context of classical Merlin-Arthur proofs 2.
} 
Our design for Theorem 1 will also rely on a polynomial extension of an algebrization of the task of clique counting. Here the $\left(\begin{array}{l}6 \\ 2\end{array}\right)$-linear algebrization is essentially due to Nešetřil and Poljak 22], but we require a substantially different algebraic circuit design to evaluate the $\left(\begin{array}{l}6 \\ 2\end{array}\right)$-linear form. In $\$ 4$ and $\$ 5$ we present a new design that nests four levels of fast matrix multiplication so that the products at the top-level multiplication can (a) be evaluated in parallel and (b) extended to a proof polynomial $P$ by careful use of Yates's algorithm 26] and fast matrix multiplication to enable efficient evaluation. The top-level multiplications rely on a trilinear representation of the matrix multiplication tensor $\langle n, n, n\rangle$ to assemble the monomials of a $\left(\begin{array}{l}6 \\ 2\end{array}\right)$-linear form out of a 3-linear base and three 4-linear parts, whose consistency is obtained via the trilinear representation. In essence, we show that 6-cliques can be counted with an arithmetic circuit of essentially the same size but with considerably more independence and depth than the Nešetřil-Poljak 22] design, which then enables the polynomial extension to a Camelot algorithm that attains the optimal speedup tradeoff relative to the Nešetřil-Poljak running time.

Our design for Theorem 2 is guided by the parallel algorithm of Björklund et al. 4 . for computing the chromatic polynomial. In particular we rely on stardard inclusionexclusion arguments 3] over tuples of independent sets, and split the domain (the vertex set) into two parts, using the ideas in [4] to sieve independent sets based on their intersections with the two parts. The key novelty in our present design is to lift the two-part approach into a univariate proof polynomial by means of Kronecker substitution, with two nested applications of Yates's algorithm to enable fast evaluation and an optimal Camelot design.

\subsection{Related work}

A Camelot algorithm seeks to simultaneously obtain essentially perfect parallelization on up to $K \leq T^{1 / 2}$ parallel nodes, a noninteractive proof of correctness (probabilistically verifiable in no more time than each parallel node individually invests into the computation), robustness against errors, and to essentially match in total running time the best known sequential algorithm for solving the problem. To our knowledge, algorithm designs simultaneously meeting these goals have not been presented earlier. However, a large body of related work exists if one relaxes one or more of the goals; the following brief overview is not exhaustive.

McConnell, Mehlhorn, Näher, and Schweitzer 21] survey certifiable algorithms that produce, together with the output, an easy-to-verify correctness proof for the output. Many works study algorithms that are resilient to errors but do not in general produce a correctness proof. For example, Caminiti, Finocchi, Fusco, and Silvetri [6] study resilient dynamic programming, Chen, Grigorescy, and de Wolf 8] study error-correcting data structures for membership queries and polynomial evaluation, Cicalese 9] studies fault-tolerant search algorithms, and Finocchi, Grandoni, and Italiano 11] present resilient sorting and searching algorithms under memory faults.

Our focus on a magicless (honest) prover is not new. Goldwasser, Kalai, and Rothblum [17 study interactive proofs with a near-linear-time verifier in the input size and with a polynomial-time prover, however without a fine-grained running time goal and without a goal for parallelizability of proof preparation.
Fraigniaud, Korman, and Peleg 12 and Göös and Suomela [16. study proofs that can be verified locally at the nodes of a network of distributed processors but without detailed consideration of the complexity to prepare such a proof.

\section{PRELIMINARIES}

This section recalls the fast arithmetic primitives underlying the Camelot framework and our highlight results.

\subsection{Fast polynomial arithmetic}

Let us recall the fast arithmetic toolbox (see von zur Gathen and Gerhard [14]) for polynomials with coefficients in a finite field $\mathbb{F}_{q}$. Computational complexity is measured in the number of arithmetic operations in $\mathbb{F}_{q}$. Multiplication and division of two polynomials of degree at most $d$ can be computed in $O(d \log d \log \log d)$ operations. The greatest common divisor of two polynomials of degree at most $d$ can be computed in $O\left(d \log ^{2} d \log \log d\right)$ operations. Given a polynomial $P(x)=p_{0}+p_{1} x+p_{2} x^{2} \ldots+p_{d} x^{d} \in$ $\mathbb{F}_{q}[x]$ and points $x_{0}, x_{1}, \ldots, x_{d} \in \mathbb{F}_{q}$ as input, the evaluation map $\left(P\left(x_{0}\right), P\left(x_{1}\right), \ldots, P\left(x_{d}\right)\right) \in \mathbb{F}_{q}^{d+1}$ can be computed in $O\left(d \log ^{2} d \log \log d\right)$ operations. Dually, given distinct points $x_{0}, x_{1}, \ldots, x_{d} \in \mathbb{F}_{q}$ and values $y_{0}, y_{1}, \ldots, y_{d} \in \mathbb{F}_{q}$ as input, the interpolation map computes the coefficients $\left(p_{0}, p_{1}, \ldots, p_{d}\right) \in \mathbb{F}_{q}^{d+1}$ of a polynomial $P(x)$ with $P\left(x_{i}\right)=y_{i}$ for all $i=0,1, \ldots, d$ in $O\left(d \log ^{2} d \log \log d\right)$ operations.

\subsection{Reed-Solomon codes}

We work with univariate polynomial codes in nonsystematic form as originally discovered by Reed and Solomon 23 to allow for fast decoding based on polynomial arithmetic (cf. Gao 15]).

Fix a finite field $\mathbb{F}_{q}$ and any $1 \leq e \leq q$ distinct elements $x_{1}, x_{2}, \ldots, x_{e} \in \mathbb{F}_{q}$. The parameter $e$ is the length of the code. To encode a message $\left(p_{0}, p_{1}, \ldots, p_{d}\right) \in \mathbb{F}_{q}^{d+1}$ consisting of $d+1$ symbols, $1 \leq d+1 \leq e$, form the message polynomial $P(x)=p_{0}+p_{1} x+p_{2} x^{2}+\ldots+p_{d} x^{d}$ and the codeword $\left(P\left(x_{1}\right), P\left(x_{2}\right), \ldots, P\left(x_{e}\right)\right) \in \mathbb{F}_{q}^{e}$. The codeword can be computed from the message in $O\left(e \log ^{2} e \log \log e\right)$ operations using fast interpolation (see 2.1.

Since a nonzero polynomial of degree $d$ has at most $d$ roots, any two codewords either agree in at most $d$ symbols or are identical. Thus, we can uniquely decode the message from any received word $\left(r_{1}, r_{2}, \ldots, r_{e}\right) \in \mathbb{F}_{q}^{n}$ that differs from the codeword in at most $(e-d-1) / 2$ symbols.

To decode the received word (or assert decoding failure), we use the following algorithm of Gao 15. As a precomputation step, compute the polynomial $G_{0}(x)=\prod_{i=1}^{e}(x-$ $\left.x_{i}\right) \in \mathbb{F}_{q}[x]$. (When $e=q$, we have $G_{0}(x)=x^{q}-x$ which suffices for our applications in this paper.) To decode $\left(r_{1}, r_{2}, \ldots, r_{e}\right) \in \mathbb{F}_{q}^{e}$, first interpolate the unique polynomial $G_{1}(x) \in \mathbb{F}_{q}[x]$ of degree at most $e-1$ with $G_{1}\left(x_{i}\right)=r_{i}$ for all $i=1,2, \ldots, e$. Second, apply the extended Euclidean algorithm to the polynomials $G_{0}(x)$ and $G_{1}(x)$, but stop as soon as the remainder $G(x)$ has degree less than $(e+d+1) / 2$. At this point suppose that $U(x) G_{0}(x)+V(x) G_{1}(x)=G(x)$. Finally, divide $G(x)$ by $V(x)$ to obtain $G(x)=P(x) V(x)+$ $R(x)$, where the degree of $R(x)$ is less than the degree of $V(x)$. If $R(x)=0$ and $P(x)$ has degree at most $d$, output $P(x)$ as the result of decoding; otherwise assert decoding failure. We observe that decoding runs in $O\left(e \log ^{2} e \log \log e\right)$ operations using fast polynomial arithmetic. 


\subsection{Yates's algorithm}

The following algorithm will be used as a subroutine of our fast evaluation algorithms in $\$ 5.3$ and $\$ 7.1$. For positive integers $s, t, k$, Yates's algorithm 26 multiplies a given $s^{k} \times 1$ vector $x$ with a structured $t^{k} \times s^{k}$ matrix $M$ to produce as output a $t^{k} \times 1$ vector $y=M x$. The structural assumption about the matrix $M$ is that it is a Kronecker power $M=A^{\otimes k}$ of a small $t \times s$ matrix $A$ with entries $\alpha_{i j}$ for $i=0,1, \ldots, t-1$ and $j=0,1, \ldots, s-1$. All arithmetic takes place in a field $\mathbb{F}$. For a positive integer $d$, let us write $[d]=\{0,1, \ldots, d-1\}$.

For positive integers $d, k$ we shall tacitly identify each $j \in$ $\left[d^{k}\right]$ with $\left(j_{1}, j_{2}, \ldots, j_{k}\right) \in[d]^{k}$. Indeed, we can view $j$ as a $k$-digit integer in base $d$, where $j_{1}, j_{2}, \ldots, j_{k}$ are the $k$ digits.

Given $x$ and $A$ as input, Yates's algorithm computes, for all $i \in\left[t^{k}\right]$, the value

$$
y_{i}=\sum_{j \in\left[s^{k}\right]} \alpha_{i_{1} j_{1}} \alpha_{i_{2} j_{2}} \cdots \alpha_{i_{k} j_{k}} x_{j}
$$

Yates's algorithm employs dynamic programming to compute the $k$ nested sums implicit in (3) one at a time. We refer to the full version of this conference abstract for a detailed exposition and extensions of Yates's algorithm. The classical version of the algorithm runs in $O\left(\left(s^{k+1}+t^{k+1}\right) k\right)$ operations, using working space for $O\left(s^{k}+t^{k}\right)$ field elements.

\section{WILLIAMS'S MERLIN-ARTHUR PRO- TOCOL FOR COUNTING $K$-CLIQUES}

This section interprets Williams's 25] Merlin-Arthur protocol for counting $k$-cliques as a Camelot algorithm. We stress that this section is for expositionary purposes only, in particular to demonstrate the Camelot framework with an easier design before proceeding to our highlight result.

\subsection{A protocol for verifying (generalized) matrix products}

Let us present a proof-polynomial version of Freivalds's classical protocol 13 to verify a matrix product and generalize it to $m$-linear products of $m>2$ matrices; such generalized matrix products have been studied e.g. by Kruskal 18.

Suppose we have $m$ integer matrices, $A^{(1)}, A^{(2)}, \ldots, A^{(m)}$, each of shape $s \times t$. We are to compute the

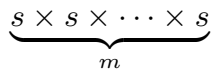

array $B$, defined for all $i \in[s]^{m}$ by

$$
b_{i}=\sum_{j=0}^{t-1} a_{i_{1} j}^{(1)} a_{i_{2} j}^{(2)} \cdots a_{i_{m} j}^{(m)}
$$

Observe that we can take $m=2$ to recover the classical matrix product $B=A^{(1)}\left(A^{(2)}\right)^{T}$ It is immediate that we can compute $B$ from the given input in $O\left(s^{m} t m\right)$ operations.

Now let us assume the role of Arthur. Suppose Merlin has computed $\tilde{B}$ (which Merlin claims to be $B$ ) and we are to check Merlin's work. Define the proof polynomial $P(x)$ as follows (5), and observe an equivalent definition (6):

$$
\begin{aligned}
P(x) & =\sum_{i \in[s]^{m}} b_{i} x^{s^{m-1} i_{m}+s^{m-2} i_{m-1}+\ldots+i_{1}} \\
& =\sum_{i \in[s]^{m}} \sum_{j=0}^{t-1} a_{i_{1} j}^{(1)} a_{i_{2} j}^{(2)} \cdots a_{i_{m} j}^{(m)} x^{s^{m-1} i_{m}+s^{m-2} i_{m-1}+\ldots+i_{1}} \\
& =\sum_{j=0}^{t-1}\left(\sum_{i_{1}=0}^{s-1} a_{i_{1} j}^{(1)} x^{i_{1}}\right) \cdots\left(\sum_{i_{m}=0}^{s-1} a_{i_{m} j}^{(m)} x^{s^{m-1} i_{m}}\right) .
\end{aligned}
$$

It is immediate that $P(x)$ has degree at most $d \leq s^{m}-1$ and that the coefficients of $P(x)$ are precisely the entries of the array $B$. Thus, in essence, by handing us (what he claims to be) $B$, Merlin has handed us the polynomial $P(x)$ in coefficient form. Using (6), without relying on Merlin's advice, we can evaluate $P\left(x_{0}\right)(\bmod q)$ at any given $x_{0} \in \mathbb{Z}_{q}$ in $O(t m s)$ operations modulo $q$. Similarly, but now relying on Merlin's advice, using (5) and Horner's rule, we can evaluate $P\left(x_{0}\right)$ $(\bmod q)$ in $O\left(s^{m}\right)$ operations. If Merlin has handed to us an incorrect $B$, we catch him with probability at least $1-d / q$ by detecting that the two evaluations disagree; recall $\$ 1.3$

\subsection{Williams's protocol for counting $k$-cliques}

Let $G$ be a graph with vertex set $[n]$ and let us assume that $k$ is an even constant. Williams's protocol counts the cliques of size $k$ in $G$ via the observation that the complete graph $K_{k}$ decomposes into two vertex-disjoint complete graphs $K_{k / 2}$ and a complete bipartite graph $K_{k / 2, k / 2}$ joining the two. The key idea is to have Merlin assist to join the two halves in all possible ways via the protocol in \$3.1. Let us assume the role of Arthur and proceed as follows. Construct a matrix $A$ of shape $n \times n^{k / 2}$ defined for all $i, j_{1}, j_{2}, \ldots, j_{k / 2} \in[n]$ by $a_{i, j_{1} j_{2} \cdots j_{k / 2}}=1$ if both (a) $j_{1}, j_{2}, \ldots, j_{k / 2}$ are distinct and form a $k / 2$-clique in $G$ and (b) $i$ is distinct from and adjacent to each of $j_{1}, j_{2}, \ldots, j_{k / 2}$ in $G$; otherwise $a_{i, j_{1} j_{2} \cdots j_{k / 2}}=0$. Have Merlin compute and send to us the

$$
\underbrace{n \times n \times \cdots \times n}_{k / 2}
$$

array $B$ defined by (4) for $s=n, t=n^{k / 2}, m=k / 2$, and $A^{(1)}=A^{(2)}=\ldots=A^{(m)}=A$. For a prime $q \geq$ $n^{k / 2}+1$, use the protocol in $\$ 3.1$ to check Merlin's work. (If the check fails, assert failure.) Take the sum of the values $b_{i}$ for all distinct $i_{1}, i_{2}, \ldots, i_{k / 2} \in\{0,1, \ldots, n-1\}$ such that $i_{1}, i_{2}, \ldots, i_{k / 2}$ form a $k / 2$-clique in $G$. This sum is $k$ ! times the number of $k$-cliques in $G$. The protocol runs in $\tilde{O}\left(n^{k / 2+1}\right)$ time.

\section{FAST AND SPACE-EFFICIENT EVALU- ATION OF A MULTILINEAR FORM}

We now proceed with the details of our highlight result, which is based on the study of a multilinear form that integrates over a function with only pairwise interactions between variables by decomposing the interactions into $\left(\begin{array}{l}6 \\ 2\end{array}\right)=$ 15 parts. The advantage of such a decomposition is that one can use fast matrix multiplication to arrive at a nontrivial arithmetic circuit for fast integration. An example application is counting small subgraphs of a graph, which we will illustrate in more detail in the next section. 


\subsection{The form and the Nešetřil-Poljak formula}

Let $\chi$ be $a \eta^{5} N \times N$ matrix over a commutative ring $\mathscr{R}$. We seek to compute the $\left(\begin{array}{l}6 \\ 2\end{array}\right)$-linear form

$$
X_{\left(\begin{array}{c}
6 \\
2
\end{array}\right)}=\sum_{\substack{a, b, c \\
d, e, f}} \chi_{a b} \chi_{a c} \chi_{a d} \chi_{a e} \chi_{a f} \chi_{b c} \chi_{b d} \chi_{b e} \chi_{b f} \chi_{c d} \chi_{c e} \chi_{c f} \chi_{d e} \chi_{d f} \chi_{e f}
$$

A direct evaluation of (7) takes $O\left(N^{6}\right)$ operations. Nešetřil and Poljak 22 observe that we can precompute the three $N^{2} \times N^{2}$ matrices

$$
\begin{aligned}
U_{a b, c d} & =\chi_{a b} \chi_{a c} \chi_{a d} \chi_{b c} \chi_{b d}, \\
S_{a b, e f} & =\chi_{a e} \chi_{a f} \chi_{b e} \chi_{b f} \chi_{e f}, \\
T_{c d, e f} & =\chi_{c d} \chi_{c e} \chi_{c f} \chi_{d e} \chi_{d f},
\end{aligned}
$$

and then use fast matrix multiplication to compute

$$
\begin{aligned}
V_{a b, c d} & =\sum_{e, f} S_{a b, e f} T_{c d, e f}, \\
X_{\left(\begin{array}{c}
6 \\
2
\end{array}\right)} & =\sum_{a, b, c, d} U_{a b, c d} V_{a b, c d} .
\end{aligned}
$$

This takes $O\left(N^{2 \omega+\epsilon}\right)$ arithmetic operations, using space for $O\left(N^{4}\right)$ scalars for any constant $\epsilon>0$.

\subsection{A new summation formula}

Our main contribution in this section is a new circuit design that matches the Nešetřil-Poljak design in the asymptotic number of arithmetic operations but reduces the space complexity from $O\left(N^{4}\right)$ to $O\left(N^{2}\right)$ scalars. In particular, the space complexity is linear in the input size. Furthermore, the new design is easily parallelizable and extendable to a proof polynomial, as we will witness in the next section.

The new design works with the following decomposition of the matrix multiplication tensor. For $r=1,2, \ldots, R$, let $\alpha_{d e}(r), \beta_{e f}(r), \gamma_{d f}(r)$ be ring elements that satisfy the multivariate polynomial identity

$$
\begin{aligned}
\sum_{d, e, f} u_{d e} v_{e f} w_{d f}=\sum_{r=1}^{R} & \left(\sum_{d, e^{\prime}} \alpha_{d e^{\prime}}(r) u_{d e^{\prime}}\right) \\
& \left(\sum_{e, f^{\prime}} \beta_{e f^{\prime}}(r) v_{e f^{\prime}}\right) \\
& \left(\sum_{d^{\prime}, f} \gamma_{d^{\prime} f}(r) w_{d^{\prime} f}\right) .
\end{aligned}
$$

We can assume that $R=O\left(N^{\omega+\epsilon / 2}\right)$ for an arbitrary constant $\epsilon>0$, where $\omega$ is the limiting exponent for the tensor rank of the matrix multiplication tensor $\langle n, n, n\rangle$ in $\mathscr{R} 5$, 19 .

The new design is as follows. For each $r=1,2, \ldots, R$,

\footnotetext{
${ }^{5}$ The present formulation admits an immediate generalization to $\left(\begin{array}{l}6 \\ 2\end{array}\right)$ distinct $N \times N$ matrices, but to keep the notation concise, we work with a single matrix $\chi$.
}

compute, using fast matrix multiplication,

$$
\begin{aligned}
& H_{a d}(r)=\sum_{e^{\prime}} \alpha_{d e^{\prime}}(r) \chi_{a e^{\prime}} \chi_{d e^{\prime}} \\
& K_{b e}(r)=\sum_{f^{\prime}} \beta_{e f^{\prime}}(r) \chi_{b f^{\prime}} \chi_{e f^{\prime}} \\
& L_{c f}(r)=\sum_{d^{\prime}} \gamma_{d^{\prime} f}(r) \chi_{c d^{\prime}} \chi_{d^{\prime} f} \\
& A_{a b}(r)=\sum_{d} \chi_{a d} \chi_{b d} H_{a d}(r), \\
& B_{b c}(r)=\sum_{e} \chi_{b e} \chi_{c e} K_{b e}(r) \\
& C_{a c}(r)=\sum_{f} \chi_{a f} \chi_{c f} L_{c f}(r) .
\end{aligned}
$$

Finally, compute, again using fast matrix multiplication,

$$
\begin{aligned}
Q_{a b}(r) & =\sum_{c} \chi_{a c} \chi_{b c} B_{b c}(r) C_{a c}(r), \\
P(r) & =\sum_{a, b} \chi_{a b} A_{a b}(r) Q_{a b}(r) .
\end{aligned}
$$

Each term $P(r)$ takes $O\left(N^{\omega+\epsilon / 2}\right)$ operations and space for $O\left(N^{2}\right)$ scalars to compute.

TheOREM 3. $X_{\left(\begin{array}{c}6 \\ 2\end{array}\right)}=\sum_{r=1}^{R} P(r)$.

Proof. Expanding and applying for each $a, b, c$ in turn, we have

$$
\begin{aligned}
\sum_{r=1}^{R} P(r)= & \sum_{r=1}^{R} \sum_{a, b, c} \chi_{a b} \chi_{a c} \chi_{b c} A_{a b}(r) B_{b c}(r) C_{a c}(r) \\
= & \sum_{r=1}^{R} \sum_{a, b, c} \chi_{a b} \chi_{a c} \chi_{b c} \sum_{d, e^{\prime}} \alpha_{d e^{\prime}}(r) \chi_{a d} \chi_{a e^{\prime}} \chi_{b d} \chi_{d e^{\prime}} \\
& \sum_{e, f^{\prime}} \beta_{e f^{\prime}}(r) \chi_{b e} \chi_{b f^{\prime}} \chi_{c e} \chi_{e f^{\prime}} \\
& \sum_{d^{\prime}, f} \gamma_{d^{\prime} f}(r) \chi_{a f} \chi_{c d^{\prime}} \chi_{c f} \chi_{d^{\prime} f} \\
= & \sum_{a, b, c} \chi_{a b} \chi_{a c} \chi_{b c} \sum_{r=1}\left(\sum_{d, e^{\prime}} \alpha_{d e^{\prime}}(r) \chi_{a d} \chi_{a e^{\prime}} \chi_{b d} \chi_{d e^{\prime}}\right) \\
& \left(\sum_{e, f^{\prime}} \beta_{e f^{\prime}}(r) \chi_{b e} \chi_{b f^{\prime}} \chi_{c e} \chi_{e f^{\prime}}\right) \\
& \left(\sum_{d^{\prime}, f} \gamma_{d^{\prime} f}(r) \chi_{a f} \chi_{c d^{\prime}} \chi_{c f} \chi_{d^{\prime} f}\right) \\
= & \sum_{a, b, c} \chi_{a b, e} \chi_{a d} \chi_{a c} \chi_{b c} \chi_{a e} \chi_{a f} \chi_{b d} \chi_{b e} \chi_{b f} \chi_{c d} \chi_{c e} \chi_{c f} \chi_{d e} \chi_{d f} \chi_{e f} .
\end{aligned}
$$

Thus, we can compute (7) from $\chi$ in $O\left(N^{2 \omega+\epsilon}\right)$ operations and space for $O\left(N^{2}\right)$ scalars. Furthermore, easy parallelization using up to $O\left(N^{\omega+\epsilon / 2}\right)$ compute nodes is now possible since the values $P(r)$ can be computed independently of each other. 


\section{A CAMELOT ALGORITHM FOR COUNTING SMALL CLIQUES}

This section proves Theorem 1. In particular, we demonstrate how to reduce counting $k$-cliques into an evaluation of the $\left(\begin{array}{l}6 \\ 2\end{array}\right)$-linear form, and then develop a proof polynomial $P(x)$ for the $\left(\begin{array}{l}6 \\ 2\end{array}\right)$-linear form, together with a fast evaluation algorithm that computes $P\left(x_{0}\right)(\bmod q)$ for given $x_{0} \in \mathbb{Z}_{q}$.

\subsection{Reduction to the multilinear form}

Suppose that 6 divides $k$. We start with a routine reduction from counting $k$-cliques in a given $n$-vertex graph $G$ to the task of evaluating the $\left(\begin{array}{l}6 \\ 2\end{array}\right)$-linear form for a particular matrix $\chi$ derived from $G$. (That is, $G$ is the common input, from which each node individually derives $\chi$.)

Let $\chi$ be an $N \times N$ matrix with $N=\left(\begin{array}{c}n \\ k / 6\end{array}\right) \leq n^{k / 6}$ and entries defined for all $A, B \in\left(\begin{array}{c}V(G) \\ k / 6\end{array}\right)$ by

$$
\chi_{A B}= \begin{cases}1 & A \cup B \text { is a clique in } G \text { and } A \cap B=\emptyset \\ 0 & \text { otherwise. }\end{cases}
$$

That is, the entries of $\chi$ indicate all pairs of $k / 6$-cliques in $G$ that together form a $k / 3$-clique.

The $\left(\begin{array}{l}6 \\ 2\end{array}\right)$-linear form $X_{\left(\begin{array}{c}6 \\ 2\end{array}\right)}$ with input $\chi$ now counts each $k$-clique in $G$ exactly $\left(\begin{array}{l}k / 6, k / 6, k / 6, k / 6, k / 6, k / 6 \\ k\end{array}\right)$ times.

\subsection{A proof polynomial for the multilinear form}

We now turn the counting algorithm in the previous section into a Camelot algorithm. First, we present a proof polynomial $P(x)$ in the Camelot framework that enables us to compute and verify the value $X_{\left(\begin{array}{c}6 \\ 2\end{array}\right)}$ from the input.

We define a univariate polynomial $P(x)$ so that we can recover $X_{\left(\begin{array}{c}6 \\ 2\end{array}\right)}$ via Theorem 3 using evaluations of $P(x)$ at integer points $x=1,2, \ldots, R$. We extend the coefficients $\alpha_{d e}(r), \beta_{e f}(r), \gamma_{d f}(r)$ into interpolation polynomials and then extend (9) and (10) into polynomials. Towards this end, for $r=1,2, \ldots, R$, let us write

$$
\Lambda_{r}(x)=\prod_{\substack{j=1 \\ j \neq r}}^{R} \frac{x-j}{r-j}
$$

for the Lagrange interpolation polynomials of degree at most $R$ for the points $1,2, \ldots, R$. The three univariate polynomials

$$
\begin{aligned}
& \alpha_{d e}(x)=\sum_{r=1}^{R} \alpha_{d e}(r) \Lambda_{r}(x), \\
& \beta_{e f}(x)=\sum_{r=1}^{R} \beta_{e f}(r) \Lambda_{r}(x), \\
& \gamma_{d f}(x)=\sum_{r=1}^{R} \gamma_{d f}(r) \Lambda_{r}(x)
\end{aligned}
$$

interpolate over the coefficients $\alpha_{d e}(r), \beta_{e f}(r), \gamma_{d f}(r)$ when $x=1,2, \ldots, R$. From (12, (9), and 10) it is immediate that the univariate polynomials

$$
\begin{aligned}
& H_{a d}(x)=\sum_{e^{\prime}} \alpha_{d e^{\prime}}(x) \chi_{a e^{\prime}} \chi_{d e^{\prime}}, \\
& K_{b e}(x)=\sum_{f^{\prime}} \beta_{e f^{\prime}}(x) \chi_{b f^{\prime}} \chi_{e f^{\prime}}, \\
& L_{c f}(x)=\sum_{d^{\prime}} \gamma_{d^{\prime} f}(x) \chi_{c d^{\prime}} \chi_{d^{\prime} f}, \\
& A_{a b}(x)=\sum_{d} \chi_{a d} \chi_{b d} H_{a d}(x), \\
& B_{b c}(x)=\sum_{e} \chi_{b e} \chi_{c e} K_{b e}(x), \\
& C_{a c}(x)=\sum_{f} \chi_{a f} \chi_{c f} L_{c f}(x),
\end{aligned}
$$

and

$$
\begin{aligned}
Q_{a b}(x) & =\sum_{c} \chi_{a c} \chi_{b c} B_{b c}(x) C_{a c}(x), \\
P(x) & =\sum_{a, b} \chi_{a b} A_{a b}(x) Q_{a b}(x) .
\end{aligned}
$$

have degree at most $3 R$ and the evaluations of $P(x)$ at $x=$ $1,2, \ldots, R$ satisfy Theorem 3 . Thus, $P(x)$ is a proof polynomial that we can employ in the Camelot framework. For the modulus $q$ we can select one or more primes $q \geq 3 R+1$ to enable interpolation and reconstruction of $X_{\left(\begin{array}{c}6 \\ 2\end{array}\right)}$ over the integers. In particular we can assume $q=O(R)$. Indeed, since $q \geq N^{2}$ and $\chi$ has entries in $\{0,1\}$, we can recover the integer $0 \leq X_{\left(\begin{array}{c}6 \\ 2\end{array}\right)}=\sum_{r=1}^{R} P(r) \leq N^{6}$ from evaluations $\sum_{r=1}^{R} P(r)$ $(\bmod q)$ for at most $O(1)$ distinct primes $q \geq 3 R+1$ using the Chinese Remainder Theorem. This gives proof size $\tilde{O}(R)$.

\subsection{The evaluation algorithm}

To complete the Camelot algorithm it remains to describe how each node evaluates, modulo $q$, the proof polynomial $P(x)$ at a given point. Let $x_{0} \in \mathbb{Z}_{q}$ be given. We seek to compute $P\left(x_{0}\right)(\bmod q)$ in time $O\left(N^{\omega+\epsilon}\right)$.

Let us assume that we have available the values $\alpha_{d e}\left(x_{0}\right)$, $\beta_{e f}\left(x_{0}\right), \gamma_{d f}\left(x_{0}\right)$ for all $d, e, f$. (This is a nontrivial assumption that we will justify in what follows.) Then, using fast matrix multiplication to evaluate (13) and (14) at $x=x_{0}$, we obtain $P\left(x_{0}\right)$ in $O\left(N^{\omega+\epsilon / 2}\right)$ operations and $O\left(N^{2}\right)$ space.

Arithmetic operations on scalars modulo $q=O(R)$ can be implemented in $\tilde{O}(1)$ time and space, which is subsumed by the bound $O\left(N^{\omega+\epsilon}\right)$. Thus, we are done assuming we can justify our earlier assumption.

To compute the values $\alpha_{d e}\left(x_{0}\right), \beta_{e f}\left(x_{0}\right), \gamma_{d f}\left(x_{0}\right)$ for all $d, e, f$, we proceed to take a look at the detailed structure of the coefficients $\alpha_{d e}(r), \beta_{e f}(r), \gamma_{d f}(r)$. We will focus on the coefficients $\alpha_{d e}(r)$, the cases for $\beta_{e f}(r)$ and $\gamma_{d f}(r)$ are symmetric.

By the properties of tensor rank and the fact that matrix multiplication tensors are closed under taking of Kronecker products, we can assume without loss of generality that $R=$ $O\left(N^{\omega+\epsilon / 2}\right)$ and that there exist positive integer constants $R_{0}, N_{0}$ and a positive integer $t$ such that $N=N_{0}^{t}$ and $R=$ $R_{0}^{t}$. In particular, we may identify the indices $d, e$ and the index $r$ with $t$-digit integers in base $N_{0}$ and $R_{0}$, respectively, so that if we write $d_{j}, e_{j}$ and $r_{j}$ for the $j$ th digit of $d, e$ 
and $r$, respectively, the coefficient $\alpha_{d e}(r)$ has the Kronecker product form

$$
\alpha_{d e}(r)=\prod_{j=1}^{t} \alpha_{d_{j} e_{j}}^{(0)}\left(r_{j}\right)
$$

where $\alpha^{(0)}$ is a matrix of size $N_{0}^{2} \times R_{0}$ with integer entries. Thus, we may view (15) as an integer matrix of size $N^{2} \times R$ which has been obtained as the $t$-fold Kronecker power of the constant-sized-and-entried matrix $\alpha^{(0)}$.

Let us now describe how to compute the $N^{2}$ coefficients $\alpha_{d e}\left(x_{0}\right)(\bmod q)$ for all $d, e$. Let us recall from $(12)$ that $\alpha_{d e}(x)$ is an interpolating polynomial for the values 15 . That is, we have, for all $d, e$,

$$
\alpha_{d e}\left(x_{0}\right)=\sum_{r=1}^{R} \alpha_{d e}(r) \Lambda_{r}\left(x_{0}\right) \quad(\bmod q) .
$$

We observe that 16 in fact describes a matrix-vector multiplication: we multiply the $N^{2} \times R$ matrix with entries $\alpha_{d e}(r)$ with the $R \times 1$ vector with entries $\Lambda_{r}\left(x_{0}\right)$. Because of the Kronecker structure 15 , this matrix-vector product can be computed in $O(R t)$ operations using Yates's algorithm ( 2.3 ).

To initialize Yates's algorithm, we require the $R \times 1$ vector, so let us start by computing the values $\Lambda_{r}\left(x_{0}\right)(\bmod q)$ for $r=1,2, \ldots, R$. If $x_{0} \in\{1,2, \ldots, R\}$ the computation is immediate (insert a 1 to position $x_{0}$ and fill the rest of the vector with $0 \mathrm{~s}$ ), so let us assume that $x_{0} \notin\{1,2, \ldots, R\}$. First, precompute the values $F_{0}, F_{1}, \ldots, F_{R-1}$ with the recurrence $F_{0}=1, \quad F_{j}=j \cdot F_{j-1}(\bmod q)$. Second, precompute the product $\Gamma\left(x_{0}\right)=\prod_{j=1}^{R}\left(x_{0}-j\right)(\bmod q)$. Finally, observe from (11) that we can compute, for each $r=1,2, \ldots, R$,

$$
\Lambda_{r}\left(x_{0}\right)=\frac{1}{(-1)^{R-r} F_{r-1} F_{R-r}} \cdot \frac{\Gamma\left(x_{0}\right)}{\left(x_{0}-r\right)}(\bmod q) .
$$

This initialization takes in total $O(R)$ operations and space modulo $q$.

Since $t=O(\log N)$ and $R=O\left(N^{\omega+\epsilon / 2}\right)$, the entire evaluation algorithm runs in $O\left(N^{\omega+\epsilon}\right)$ time and space. This completes the Camelot framework for computing and verifying $X_{\left(\begin{array}{c}6 \\ 2\end{array}\right)}$ and thus proves Theorem 1

\section{A PROOF TEMPLATE FOR PARTITIONING SUM-PRODUCTS}

This section supplies a template for proof polynomials that underlies our highlight result for the chromatic polynomial. We rely on Kronecker substitution to enable succinct univariate encoding of the partitioning property.

\subsection{The problem}

Let $U=\{1,2, \ldots, n\}$ be an $n$-element ground set (or universe), and let us write $2^{U}$ for the set of all subsets of $U$. Let us call a function $f: 2^{U} \rightarrow \mathbb{Z}$ a set function.

Suppose the common input consists of at least $n$ bits, known to all the nodes. Suppose the input defines a set function $f$ such that for any given $X \subseteq U$ any node can compute the value $f(X)$ in time $O^{*}(1)$. Furthermore, we assume that based on the input each node can in $O^{*}(1)$ time compute an upper bound $\phi$ such that $|f(X)| \leq \phi$ for all $X \subseteq U$.
For a positive integer $t$ with $t=O^{*}(1)$, the nodes would like to compute the value of the $t$-part partitioning sum product

$$
\sum_{\left(X_{1}, X_{2}, \ldots, X_{t}\right)} f\left(X_{1}\right) f\left(X_{2}\right) \cdots f\left(X_{t}\right),
$$

where the sum is over all $t$-tuples $\left(X_{1}, X_{2}, \ldots, X_{t}\right) \in 2^{U} \times$ $2^{U} \times \cdots \times 2^{U}$ such that

$$
\begin{aligned}
& X_{1} \cup X_{2} \cup \cdots \cup X_{t}=U \\
& \quad \text { and } X_{i} \cap X_{j}=\emptyset \text { holds for all } 1 \leq i<j \leq t .
\end{aligned}
$$

That is, the $t$-tuple $\left(X_{1}, X_{2}, \ldots, X_{t}\right)$ partitions the universe $U$ into $t$ pairwise disjoint parts $X_{1}, X_{2}, \ldots, X_{t}$ so that the ordering of the parts is relevant and zero or more empty parts are permitted. On its own, a node can compute 17 in time $O^{*}\left(2^{n}\right)$ [3].

\subsection{The proof polynomial}

This section defines the proof polynomial $P(x)$ for partitioning sum-products. We start by partitioning the universe $U$ into two disjoint parts, $U=E \cup B$, where $E$ is the explicit set (whose subsets each node will track explicitly) and $B$ is, by slight abuse of terminology, a set of bits (where the node will rely on advice from the other nodes).

Let us assume that the set $B$ of bits is $\left\{0,1, \ldots, 2^{|B|-1}\right\}$. With this assumption, select exactly $|B|$ bits, possibly with repetition. Observe that if your bits sum to $2^{|B|}-1$, then you have selected each bit exactly once (without repetition).

Let us also recall that there are exactly $\left(\begin{array}{c}m+k-1 \\ k-1\end{array}\right)$ ways to place $m$ identical balls into $k$ distinct bins. Put otherwise, we can form exactly $\left(\begin{array}{c}m+k-1 \\ k-1\end{array}\right)$ distinct multisets of size $m$ over a ground set of $k$ elements. Let us write $\left(\left(\begin{array}{l}B \\ k\end{array}\right)\right)$ for the set of multisets of size $k$ over $B$. In particular, $\left|\left(\left(\begin{array}{c}B \\ |B|\end{array}\right)\right)\right|=\left(\begin{array}{c}2|B|-1 \\ |B|-1\end{array}\right)$ is precisely the number of ways we can select $|B|$ bits out of $B$, possibly with repetition. Let us write $\sum M$ for the sum of elements in $M \in\left(\left(\begin{array}{c}B \\ |B|\end{array}\right)\right)$. With this notation, our previous observation is equivalent the statement that for all $M \in\left(\left(\begin{array}{c}B \\ |B|\end{array}\right)\right)$ we have $\sum M=2^{|B|}-1$ if and only if $M=B$.

It will be convenient to present the proof polynomial first over the integers, and only then choose appropriate primes $q$. The proof polynomial is

$$
P(x)=p_{0}+p_{1} x+p_{2} x^{2}+\ldots+p_{d} x^{d}
$$

whose coefficients are defined for all $s=0,1, \ldots, d$ with $d=2^{|B|-1}|B|$ by

$$
p_{s}=\sum_{\left(X_{1}, X_{2}, \ldots, X_{t}\right)} f\left(X_{1}\right) f\left(X_{2}\right) \cdots f\left(X_{t}\right),
$$

where the sum is over all $t$-tuples $\left(X_{1}, X_{2}, \ldots, X_{t}\right) \in 2^{U} \times$ $2^{U} \times \cdots \times 2^{U}$ that satisfy the multiset equality

$$
X_{1}+X_{2}+\ldots+X_{t}=E+M
$$

for a multiset $M \in\left(\left(\begin{array}{c}B \\ |B|\end{array}\right)\right)$ with $\sum M=s$. (We use additive notation for multisets to stress that the element multiplicities must agree for each element in the ground set $U$.)

We observe that $p_{2|B|-1}$ agrees with (17). Indeed, (18) holds if and only if (21) holds with $B=M$. Furthermore, $B=M$ if and only if $\sum M=2^{|B|-1}$. Thus, each node needs to get confidence that the coefficients $p_{0}, p_{1}, \ldots, p_{d}$ have been correctly computed, over the integers. 
Since $P(x)$ has degree at most $2^{|B|-1}|B|$, it suffices to use primes $q$ with $q=O^{*}\left(2^{|B|}\right)$ to enable evaluation and reconstruction of the proof modulo $q$. Such primes can be found in time $O^{*}(1)$ 1].

Each node can use the upper bound $\phi$ on the absolute values of $f$ to compute in time $O^{*}(1)$ the upper bound $2^{n t+1} \phi^{t}$ for the absolute values in 20 . Assuming that $\log q=\Omega(n)$ and $\log q=\Omega(\phi)$, which will be the case in our instantiations of the template, it suffices to work with $O^{*}(1)$ distinct primes $q$ to reconstruct the integer coefficients $p_{0}, p_{1}, \ldots, p_{d}$. Indeed, we have $t=O^{*}(1)$ and $\log \phi=O^{*}(1)$ since $\phi$ is computable from the common input in time $O^{*}(1)$.

\subsection{A template for the evaluation algorithm}

Let $x_{0} \in\{0,1, \ldots, q-1\}$ be given. We now describe how a node computes $P\left(x_{0}\right)(\bmod q)$, but will leave one implementation detail unspecified, namely how the function $g$ is computed within the desired computational budget, with the understanding that such algorithms will be provided in subsequent sections when the template is instantiated. By "computing a function" we mean computing a table that contains the value of the function for every possible input to the function.

Let $w_{E}, w_{B}$ be formal indeterminates. That is, each node will be computing with polynomials in $w_{E}, w_{B}$ with integer coefficients normalized to $\{0,1, \ldots, q-1\}$ modulo $q$. Each node computes the function $g: 2^{E} \rightarrow \mathbb{Z}_{q}\left[w_{E}, w_{B}\right]$ defined for all $Y \subseteq E$ by

$$
g(Y)=\sum_{\substack{X \subseteq U \\ X \cap \bar{E} \subseteq Y}} f(X) w_{E}^{|X \cap E|} w_{B}^{|X \cap B|} \prod_{b \in X \cap B} x_{0}^{b}(\bmod q) .
$$

(Recall that $B=\left\{0,1, \ldots, 2^{|B|-1}\right\}$ so the expression $x_{0}^{b}$ is well-defined.) The time budget for computing the function $g$ is $O^{*}\left(2^{|E|}\right)$.

Assuming the node has computed $g$, it next computes the polynomial

$$
\begin{aligned}
a\left(w_{E}, w_{B}\right) & =\sum_{j_{E}, j_{B}} a_{j_{E}, j_{B}} w_{E}^{j_{E}} w_{B}^{j_{B}} \\
& =\sum_{Y \subseteq E}(-1)^{|E \backslash Y|} g(Y)^{t} \quad(\bmod q) .
\end{aligned}
$$

The node is interested on $a_{|E|,|B|}$, the integer coefficient of the monomial $w_{E}^{|E|} w_{B}^{|B|}$ in $a(w)$. Using 23 and the computed $g$, the node can compute $a_{|E|,|B|}$ in time $O^{*}\left(2^{|E|}\right)$.

From 22, 23), and standard inclusion-exclusion arguments 3 it follows that, modulo $q$,

$$
a_{|E|,|B|}=\sum_{\left(X_{1}, X_{2}, \ldots, X_{t}\right)} f\left(X_{1}\right) f\left(X_{2}\right) \cdots f\left(X_{t}\right) \prod_{j=1}^{t} \prod_{b \in X_{j} \cap B} x_{0}^{b}
$$

where the sum is over all $t$-tuples $\left(X_{1}, X_{2}, \ldots, X_{t}\right) \in 2^{U} \times$ $2^{U} \times \cdots \times 2^{U}$ for which there exists a multiset $M \in\left(\left(\begin{array}{c}B \\ |B|\end{array}\right)\right)$ with

$$
X_{1}+X_{2}+\ldots+X_{t}=E+M .
$$

Furthermore, every multiset $M \in\left(\left(\begin{array}{c}B \\ |B|\end{array}\right)\right)$ satisfies $\sum M \in$ $\left\{0,1, \ldots, 2^{|B|-1}|B|\right\}$.

Comparing 20 and 21 with 24 and 25, we have

$$
P\left(x_{0}\right)=a_{|E|,|B|} \quad(\bmod q) .
$$

That is, we have evaluated the polynomial $P(x)$ at the chosen point $x_{0}$ modulo $q$.

\subsection{Running time for evaluation}

Assuming the node keeps to its budget for computing the function $g$, we observe that $P\left(x_{0}\right)(\bmod q)$ for a given $x_{0} \in$ $\{0,1, \ldots, q-1\}$ can be computed in time $O^{*}\left(2^{|B|}+2^{|E|}\right)$. This is minimized when $|B|=|E|$. Since $n=|U|=|E|+|B|$, we have $|E|=|B|=n / 2$.

\section{THE CHROMATIC POLYNOMIAL}

We instantiate the template in $\$ 6$ for the chromatic polynomial. Let $G$ be an undirected graph with $n$ vertices. The chromatic polynomial $\chi_{G}(t)$ is a polynomial in $t$ of degree at most $n$. Thus the values of $\chi_{G}(t)$ at any $n+1$ points suffice to reconstruct $G$ by interpolation.

The value $\chi_{G}(t)$ for a positive integer $t=1,2, \ldots, n+1$ counts the number of mappings $c: V(G) \rightarrow\{1,2, \ldots, t\}$ such that $c(u) \neq c(v)$ holds for all edges $\{u, v\} \in E(G)$. Such mappings are in a bijective correspondence with the $t$-tuples $\left(X_{1}, X_{2}, \ldots, X_{t}\right)$ that partition $U=V(G)$ into $t$ pairwise disjoint parts $X_{1}, X_{2}, \ldots, X_{t}$ such that each $X_{i} \subseteq U$ is an independent set of $G$.

Thus, $\chi_{G}(t)$ equals the partitioning sum-product 17 if we choose the set function $f: 2^{U} \rightarrow \mathbb{Z}$ to be the indicator function for independent sets in $G$. That is, for all $X \subseteq U$ we define

$$
f(X)= \begin{cases}1 & \text { if } X \text { is independent in } G ; \\ 0 & \text { otherwise. }\end{cases}
$$

In particular, we can take $\phi=1$ and use the template for $t=1,2, \ldots, n+1$ to interpolate the polynomial $\chi_{G}(t)$ from $\chi_{G}(1), \chi_{G}(2), \ldots, \chi_{G}(n+1)$.

\subsection{Computing the node function}

Suppose that $t=1,2, \ldots, n+1$ is fixed. It remains to complete the template for evaluating the function 22 with $f$ defined by 26 in time $O^{*}\left(2^{n / 2}\right)$. In particular, we instantiate the template with $|E|=|B|=n / 2$.

Computing 22 within the time budget requires a dedicated algorithm since $G$ may have up to $2^{n}$ independent sets, and hence the sum 22 may have up to $2^{n}$ terms (for $Y=E)$, which is well above our time budget.

The intuition that we pursue in what follows is that an arbitrary independent set $I \subseteq E \cup B$ in $G$ remains independent in $G$ when restricted to the induced subgraphs $G[E]$ and $G[B]$. Furthermore, $I \cap B$ is disjoint from the neighborhood of $I \cap E$ in $B$ but otherwise has no interactions with $I \cap E$. These observations enable us to compute 22 in parts across the cut $(E, B)$ with the help of Yates's algorithm $(2.3)$.

The node proceeds as follows. Let $q$ and $x_{0}$ be as in the template. First, the node computes the function $\hat{f}_{B}: 2^{B} \rightarrow$ $\mathbb{Z}_{q}\left[w_{E}, w_{B}\right]$ defined, modulo $q$, for all $X \subseteq B$ by

$$
\hat{f}_{B}(X)= \begin{cases}\prod_{b \in X} w_{B} x_{0}^{b} & \text { if } X \text { is independent in } G ; \\ 0 & \text { otherwise. }\end{cases}
$$

This takes time $O^{*}\left(2^{|B|}\right)$. Next, using Yates's algorithm, the node computes in time $O^{*}\left(2^{|B|}\right)$ the function $g_{B}: 2^{B} \rightarrow$ $\mathbb{Z}_{q}\left[w_{E}, w_{B}\right]$ defined for all $Y \subseteq B$ by

$$
g_{B}(Y)=\sum_{X \subseteq Y} \hat{f}_{B}(X) \quad(\bmod q) .
$$


For a subset $X \subseteq E$, let us write $\Gamma_{G, B}(X)$ for the set of all vertices $v \in B$ that have at least one neighbor in the set $X$ in $G$. Next, using the function $g_{B}$ that the node has computed, it computes the function $\hat{f}_{E}: 2^{E} \rightarrow \mathbb{Z}_{q}\left[w_{E}, w_{B}\right]$ defined, modulo $q$, for all $X \subseteq E$ by

$$
\hat{f}_{E}(X)= \begin{cases}w_{E}^{|X|} g_{B}\left(B \backslash \Gamma_{G, B}(X)\right) & \text { if } X \text { is ind. in } G ; \\ 0 & \text { otherwise. }\end{cases}
$$

Remark. The term $g_{B}\left(B \backslash \Gamma_{G, B}(X)\right)$ in 29 extends an independent set $X \subseteq E$ with contributions from independent sets in $B$ in all possible ways that are compatible with $X$. This aggregation of contributions across the cut $(E, B)$ is the key to staying within the time budget $O^{*}\left(2^{n / 2}\right)$.

Finally, the node computes the function $g$ in time $O^{*}\left(2^{|E|}\right)$ using the function $\hat{f}_{E}$ and Yates's algorithm. Indeed, comparing (22) and (26) with (27), 28), and (29), we observe that

$$
g(Y)=\sum_{X \subseteq Y} \hat{f}_{E}(X) \quad(\bmod q)
$$

\section{Acknowledgments}

We thank Ryan Williams for useful discussions and giving us early access to his manuscript describing his batch evaluation framework 25 of which the present "Camelot" framework forms a special case. We also thank Stefan Schneider and Russell Impagliazzo for an inspiring exposition of the nondeterministic exponential time hypothesis and the Carmosino et al. 7] results at the Simons Institute in fall 2015. Finally, we thank Jukka Suomela and the anonymous referees for useful comments.

\section{REFERENCES}

[1] M. Agrawal, N. Kayal, N. Saxena, PRIMES is in P, Ann. of Math. (2) 160 (2004) 781-793.

[2] L. Babai, S. Moran, Arthur-Merlin games: a randomized proof system, and a hierarchy of complexity classes, J. Comput. System Sci. 36 (1988) 254-276.

[3] A. Björklund, T. Husfeldt, M. Koivisto, Set partitioning via inclusion-exclusion, SIAM J. Comput. 39 (2009) 546-563.

[4] A. Björklund, T. Husfeldt, P. Kaski, M. Koivisto, Covering and packing in linear space, Inform. Process. Lett. 111 (2011) 1033-1036.

[5] P. Bürgisser, M. Clausen, M. A. Shokrollahi, Algebraic Complexity Theory, Springer, 1997.

[6] S. Caminiti, I. Finocchi, E. G. Fusco, F. Silvestri, Resilient dynamic programming, Algorithmica, to appear.

[7] M. L. Carmosino, J. Gao, R. Impagliazzo, I. Mihajlin, R. Paturi, S. Schneider, Nondeterministic extensions of the strong exponential time hypothesis and consequences for non-reducibility, Proc. 2016 ACM Conference on Innovations in Theoretical Computer Science (ITCS'16, Cambridge, MA, January 14-16, 2016), ACM, 2016, pp. 261-270.

[8] V. Chen, E. Grigorescu, R. de Wolf, Error-correcting data structures, SIAM J. Comput. 42 (2013) 84-111.

[9] F. Cicalese, Fault-Tolerant Search Algorithms, Springer, Heidelberg, 2013.
[10] F. Eisenbrand, F. Grandoni, On the complexity of fixed parameter clique and dominating set, Theoret. Comput. Sci. 326 (2004) 57-67.

[11] I. Finocchi, F. Grandoni, G. F. Italiano, Optimal resilient sorting and searching in the presence of memory faults, Theoret. Comput. Sci. 410 (2009) 4457-4470.

[12] P. Fraigniaud, A. Korman, D. Peleg, Towards a complexity theory for local distributed computing, J. ACM 60 (2013), no. 5, Art. 35.

[13] R. Freivalds, Probabilistic machines can use less running time, IFIP Congress (1977) 839-842.

[14] J. von zur Gathen, J. Gerhard, Modern Computer Algebra, 3rd ed., Cambridge University Press, 2013.

[15] S. Gao, A new algorithm for decoding Reed-Solomon codes, in: V. K. Bhargava, H. V. Poor, V. Tarokh, S. Yoon, eds., Communications, Information, and Network Security, Springer, 2003, pp. 55-68.

[16] M. Göös, J. Suomela, Locally checkable proofs, Proc. 30th Annual ACM Symposium on Principles of Distributed Computing (PODC'11, San Jose, CA, June 6-8, 2011), ACM, 2011, pp. 159-168.

[17] S. Goldwasser, Y. T. Kalai, G. N. Rothblum, Delegating computation: Interactive proofs for muggles, J. ACM 62 (2015) Art. 27.

[18] J. B. Kruskal, Three-way arrays: rank and uniqueness of trilinear decompositions, with application to arithmetic complexity and statistics, Linear Algebra and Appl. 18 (1977) 95-138.

[19] J. M. Landsberg, Tensors: Geometry and Applications, American Mathematical Society, 2012.

[20] F. Le Gall, Powers of tensors and fast matrix multiplication, Proc. International Symposium on Symbolic and Algebraic Computation (ISSAC'14, Kobe, July 23-25, 2014), ACM, 2014, pp. 296-303.

[21] R. M. McConnell, K. Mehlhorn, S. Näher, P. Schweitzer, Survey: Certifying algorithms, Computer Science Review 5 (2011) 119-161.

[22] J. Nešetřil, S. Poljak, On the complexity of the subgraph problem, Comment. Math. Univ. Carolin. 26 (1985) 415-419.

[23] I. S. Reed, G. Solomon, Polynomial codes over certain finite fields, J. Soc. Indust. Appl. Math. 8 (1960) 300-304.

[24] D. Tiwari, S. Gupta, G. Gallarno, J. Rogers, D. Maxwell, Reliability lessons learned from GPU experience with the Titan supercomputer at Oak Ridge leadership computing facility,

Proc. International Conference for High Performance Computing, Networking, Storage and Analysis (SC'15, Austin, TX, November 15-20, 2015), Art. 38.

[25] R. Williams, Strong ETH breaks with Merlin and Arthur: Short non-interactive proofs of batch evaluation, Proc. 31st IEEE Colloquium on Computational Complexity (CCC'16, Tokyo, May 29-June 1, 2016), to appear.

[26] F. Yates, The Design and Analysis of Factorial Experiments, Imperial Bureau of Soil Science, Harpenden, 1937. 